\title{
Atomistic simulation of shape memory effect (SME) and superelasticity (SE) in nano-porous NiTi shape memory alloy (SMA)
}

\author{
Sourav Gur ${ }^{1}$, George N. Frantziskonis ${ }^{1,2, *}$ and Krishna Muralidharan ${ }^{2}$
}

\author{
${ }^{1}$ Civil Engineering and Engineering Mechanics, University of Arizona, Tucson, AZ 85721 USA \\ ${ }^{2}$ Materials Science and Engineering, University of Arizona, Tucson, AZ 85721 USA \\ *Corresponding author Email: frantzis@email.arizona.edu (G. N. Frantziskonis)
}

\begin{abstract}
Porosity can play an important role in altering the phase transformation characteristics of NiTi shape memory alloys (SMA), thus changing its shape memory as well as its superelasticity properties. This work, based on atomistic simulations of binary NiTi SMA, documents the effects of porosity at the nanometer length scale on phase fraction evolution kinetics, transformation temperatures, and stress-strain response. Classical molecular dynamics simulations are performed using a well-examined and verified Finnis-Sinclair type embeddedatom method interatomic potential. Simulation results for the nano-porous NiTi with various porosity configurations are compared to non-porous NiTi. The martensite phase fraction and transformation temperatures increase noticeably with increasing porosity, and the stress-strain response shows noticeable variation with porosity. The residual strain and hysteretic energy dissipation capacity increase significantly with increasing porosity.
\end{abstract}

Keywords: Atomistic simulation, Shape memory effect, Superelasticity, NiTi shape memory alloy, nanoscale, porosity

\section{Introduction}

Important properties of NiTi shape memory alloy (SMA) emanating from the temperature and stress or strain induced displacive phase transformation process are the shape memory effect (SME) and superelasticity (SE). While the SME relates to the thermally induced phase transformation process, the SE relates to the stress or strain-controlled phase transformation process at different temperatures. Because of its tunable and well characterized material properties, NiTi SMA has become one of the alternative and attractive choice for research as well as for industrial applications. Review papers [1-3] and other recent studies [4-7] report investigations on novel smart components and devices at various length scales, from nanometer to continuum, using NiTi SMAs and/or relevant composites. The SME and SE properties can be altered and/ or tuned through tailoring the evolution of microstructure, i.e., the evolution kinetics of different phases and this leads to a broader spectrum of applications of NiTi SMA.

Microstructure of NiTi SMA can be tailored via for example altering its composition or by the introduction of free surfaces/interfaces through porosity. Based on previous studies [5-10], tailoring the microstructure can alter the: (i) evolution and growth/nucleation kinetics of different phases; (ii) phase transformation temperatures and the stress/strain response. Different studies show that changing the $\mathrm{Ni} / \mathrm{Ti}$ fraction, and/or adding other elements (such as in ternary $\mathrm{Ni}-\mathrm{Ti}-\mathrm{X}(\mathrm{X}=\mathrm{Cr}, \mathrm{Cu}, \mathrm{Hf}, \mathrm{Pd}, \mathrm{V}, \mathrm{Zr})$ and quaternary Ni-Ti-Cu-Y (Y = Co, Pd) SMAs, etc.) 
can significantly alter the phase transformation temperatures, critical energy or stress required for phase transformation, and the width of the thermal and/or stress hysteresis. Recent studies by the authors and others at the nanometer length scale [4-10] demonstrate the significant effect of free surfaces and interfaces on the phase fraction evolution, transformation temperatures and stress-strain response. Increase in available surface area supports some unique behavior in the phase transformation process [4-10], which may not present in non-porous NiTi. Similarly, porosity is an alternative way to tailor the microstructure evolution process, and thereby altering the SME and SE properties of NiTi SMA.

Porous SMAs, the most common one being NiTi, have been studied extensively, starting in the 1980s with applications in the area of biomedical engineering. Production methods for porous NiTi vary widely, yet the basic processes fall under the general category of powder metallurgy. The extensive literature in this area, including physical testing and characterization of porous NiTi can be traced through a recent review [11]. In parallel, extensive efforts on modeling the thermomechanical behavior of SMAs in general and NiTi in particular have also been undertaken. Unit cell and spatial averaging modeling methods (based on mean field method for homogenization or micromechanical averaging method) [12-16] yield effective macroscopic constitutive relations including the evolution of phase fractions in the SMA. Also, the effects of hydrostatic pressure, asymmetry of the constitutive response between tension and compression, are included in the model presented in [17]. Numerical modeling of SMAs, based on the phenomenological constitutive relation are reported in the literature [18-23]. Other mesoscopic/ microscopic models for porous NiTi include those reported in [24,25], and some of them are developed from phase-field theory [26,27]. Significant level of experimental studies [28-31] are also reported that explore the phase transformation and superelasticity property of SMA, under a wide range of constant and gradient porosity.

From numerous studies it has been observed that the key to the behavior of $\mathrm{NiTi}$, and thus of porous $\mathrm{NiTi}$, is its phase transformation kinetics, and such kinetics depend on spatial scale $[9,10]$. It has been observed from previous studies $[4,7-10]$ that, at the nanoscale, the presence of free surfaces, substantially alters the phase transformation kinetics of NiTi. Free surfaces act as defects that initiate the forward and reverse phase-transformation process involving the different variants of the martensite phase and the austenite phase respectively. However, such kinetics, and their effects on the SME and SE of porous NiTi, have not been studied at the nanometric scale, and this creates a gap of knowledge about the behavior of porous SMAs, at the nanometer length scale. Thus, this study addresses the nanoscale porous NiTi, of various porosity configurations, computationally, and compares the results to available experimental data.

Specifically, the present study addresses the effects of porosity on the SME and SE behavior of NiTi SMA. Porous NiTi cells of various levels of porosity are simulated using an interatomic potential that is has been well-documented for its effectiveness in simulating NiTi SMA. The effects of porosity on the temperature and stress/ strain induced phase transformation process is reported in terms of martensite phase fraction, transformation temperatures, and width of the thermal hysteresis. Also, the effects of porosity on the stress-strain response, residual strain and hysteresis energy dissipation capacity of NiTi SMA at various temperatures are reported. 


\section{Methods}

This section provides the information about the interatomic potential, MD simulation process in LAMMPS [32,33] and on the characterization techniques used to separate the spatial domain of austenite phase from that of martensite. A brief description of the simulation methods is given since they are well-established and described in details in the literature $[9,10,34]$.

\subsection{Interatomic potential}

In the present study, a well-established many-body Finnis-Sinclair type interatomic potential (as referred by the embedded-atom method (EAM) in literature) for the binary NiTi alloy is used. The EAM potential was originally developed by Lai and Liu [35] considering the secondmoment approximation of tight-binding theory (TBSMA); and later improved by Zhong et al $[6,36,37]$ through adding a smooth cutoff behavior (to avoid the diverging forces during simulation, due to large atomic displacements) above a critical interatomic distance [6,36,37]. Different parameters of this EMA potential are estimated by fitting the properties (obtained from first principles calculations) of the B2 phase at $0 \mathrm{~K}$, with the potential cut off radius $r_{c}=$ $4.2 \AA[6,36,37]$. It has been shown in several studies [6,7,9,10,34,36,37] that this modified potential provides accurate values of the lattice constants and energies of different phases of NiTi, when compared to those obtained from ab-initio calculations [36,37]. Further, other studies confirm that this modified potential predicts appropriately (i) the phase transformation (PT) process due to the temperature i.e. shape memory effect (SME) and super-elasticity (SE) [4,7-10,36,37], (ii) transformation temperatures and stress values [4,6,8,9,34,36,37], and the phase fraction evolution kinetics and the stress-strain responses [4,6,8,9,34,37]. Since this potential is well documented in the literature [6,7,9,10,34,36,37], further details are not provided here; rather, a brief description is provided in the supplementary material.

\subsection{MD simulation process}

In this study both the shape memory effect (SME) and super-elasticity (SE) aspects of nanoporous NiTi are explored computationally and the results are compared to non-porous ones. In all MD simulations, the initial MD cell size is $250 \AA \times 250 \AA \times 250 \AA$, with periodic boundary conditions in all three directions.

To generate porous MD simulation cells, a MD visualization and rendering software - OVITO [38] is used. Figure 1 (a) shows a typical MD simulation cell corresponding to a nonporous B2 NiTi system as rendered using OVITO. To generate porous MD cells, spherical domains of different diameters (ranging from $10 \AA$ to $50 \AA$ ) are identified within the non-porous structure (using OVITO), and equal number of $\mathrm{Ni}$ and $\mathrm{Ti}$ are atoms are removed from within this sphere.

For all simulations, the pristine simulation cells are initially geometrically optimized through the stress-controlled conjugate gradient energy minimization method. At each temperature of interest, the system is initially subjected to NPT (i.e. constant pressure $=0 \mathrm{GPa}$ ) conditions for 360 ps, where the shape and size of the simulation boxes are allowed to change to ensure that both pressure as well as the individual components of the symmetric stress tensor are set to zero. This is followed by constant volume (NVT) conditions for an additional 240 ps. In each 
case of NPT or NVT stabilization, the adopted time step is $\Delta t=0.0005$ ps. During the simulation process, the temperature and pressure is controlled by a Nose-Hoover thermostat and barostat. In order to remove the effects of thermal fluctuations while analyzing structural characteristics (carried out during the NVT stage), the atomic coordinates are averaged over 20 ps. It is verified that the systems are well-equilibrated and converged during both NPT and NVT simulations. For this, the fluctuations (with respect to the mean values) in total energy and temperature are kept smaller than $0.001 \mathrm{eV} /$ atom and $0.50 \mathrm{~K}$, respectively.
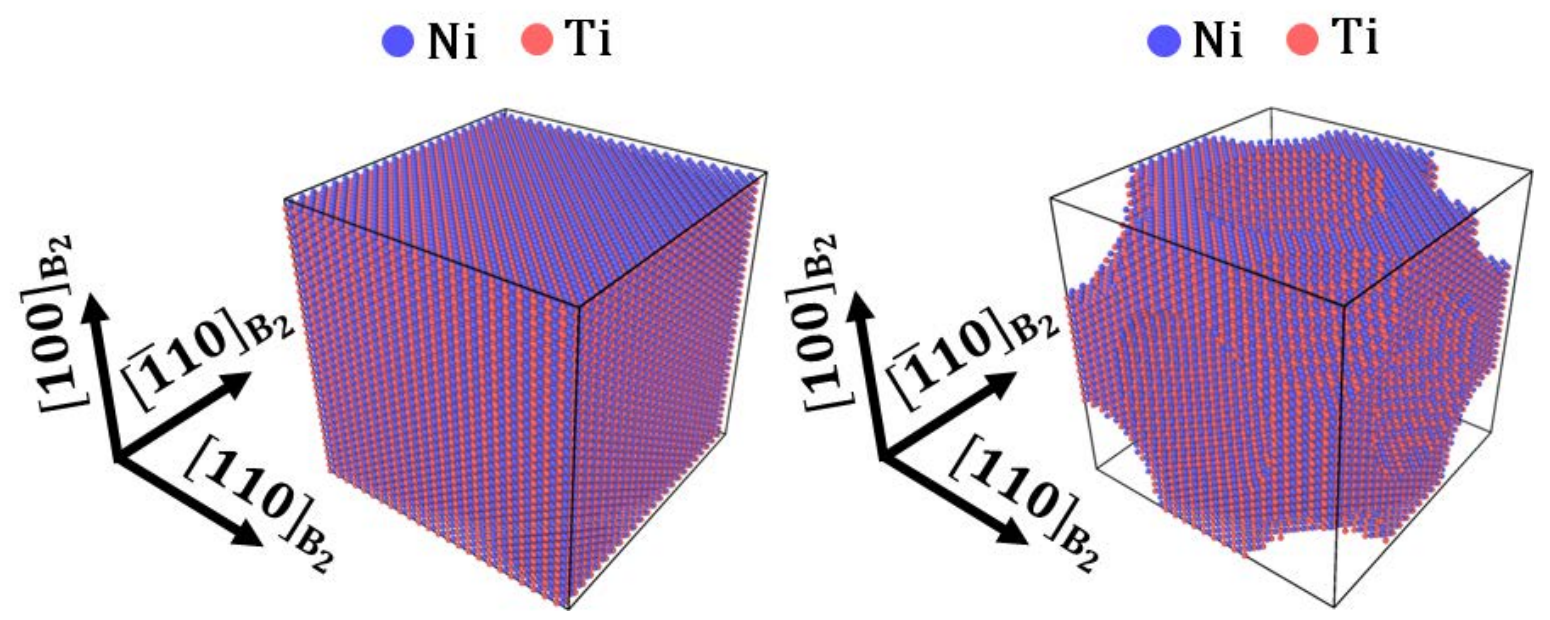

Figure-1: MD simulation cell corresponding to (a) non-porous NiTi and (b) porous NiTi. Periodic boundaries are imposed ensuring that the pores at the cell-boundaries are continuous.

\subsubsection{Thermally induced phase transformation process and SME}

MD simulations of porous and non-porous NiTi SMA are performed within the temperature range of $50-700 \mathrm{~K}$. Consideration of such temperature range is important for the accurate estimation of transformation temperatures and phase fractions. Within the phase transformation temperature regime (250-450 K), simulations are performed at an interval of $10 \mathrm{~K}$ temperature, while outside this temperature range, $50 \mathrm{~K}$ interval is considered. Initially, for the thermally induced martensitic (i.e. austenite to martensite) and austenitic (i.e. martensite to austenite) phase transformation process, pristine B2 (with the crystallographic orientation [110], [11̄0] and [001]) and pristine B19' (with the crystallographic orientation [100], [010] and [001]) supercells are adopted, respectively. The MD simulations are performed using the above mentioned stabilization process under constant and equal to zero pressure.

\subsubsection{Stress/strain induced phase transformation process and SE}

To study the SE in porous and non-porous NiTi SMA, equilibrated supercells at different temperatures ( $\mathrm{T}=250,300$ 350, 400 and $450 \mathrm{~K}$ ) are considered, and uniaxial stress (tension) controlled simulations are performed. The tensile loading/ unloading cycle is discretized into 25 steps and in every step, temperature and pressure are controlled by a Nose-Hoover thermostat and barostat. Tensile stress (along the loading and unloading direction) on the simulation box/supercell is incrementally increased (or decreased for unloading) in steps, while the stress in all other directions are kept at zero. In every step of loading/unloading, initially, the thermally equilibrated periodic B2 or B19' simulation boxes are considered and the above 
mentioned stabilization process is applied. During the forward/martensitic transformation process (i.e., under loading), tensile stress is applied along the $\langle 110\rangle_{\mathrm{B} 2}$ direction. While, in the reverse/ martensitic transformation process (i.e., under unloading), tensile stress is applied along the $\langle 100\rangle_{\mathrm{B} 19^{\prime}}$ direction, which corresponds to the martensitic phase transformation direction of B2, i.e. the $\langle 110\rangle_{\mathrm{B} 2}$ direction.

\subsection{Structural characterization}

To distinguish austenite B2 phase from the martensite B19' phase variants, the bond length based order parameter proposed by Mutter and Nielaba $[4,8]$ is adopted. According to this order parameter, a pristine B2 and B19' structure has a value of -1.0 and +1.0, respectively. However, the order parameter shows a narrow Gaussian distribution centering around -1.0 and +1.0 for the B2 and B19' phases, respectively. This uncertainty in the bond order parameter corresponds to fluctuations in the lattice parameters of B2 and B19' phases, due to the combined effects of linear thermal expansion $(\alpha \Delta T)$, interface effects, and internal strains $\left(\varepsilon^{*}\right)$ that develop during the phase transformation process. Based on the data from all performed simulations, it is observed that the order parameters for all B19' phase atoms are greater than +0.95 , and for all B2 phase atoms are less than -0.95 . Thus, in this study, atoms with order parameter greater than +0.95 correspond to martensite B19' phase and those less than -0.95 to austenite B2 phase. During simulation, other than the austenite B2 and martensite B19' phases, two other stable variants of martensite B19 is observed and characterized using this order parameter. Here also, for B19 phase, the order parameter shows a narrow Gaussian distribution centering at \pm 0.16 (for two different variants). B19 variants are only observed in porous NiTi. As reported in other MD simulation studies [7,9,10,34], metastable B19 can only be stabilized due to the presence of surfaces, and thus only observed in porous NiTi, not in non-porous NiTi. Further details on the bond length based order parameter and evolution and estimation of different phases can be found in $[4,7-10,34]$ and in the supplementary material.

\section{Results and discussion}

The present study shows the effects of porosity on the shape memory effect (SME) and superelastic (SE) of NiTi at the nanometer scale. Pores in NiTi are introduced through noninterconnected spherical inclusions. The adopted diameter of the spherical inclusions is within the range of $10 \AA$ to $50 \AA$. Introduction of pores creates internal surfaces, which increase with increasing porosity. Previous studies $[5-7,9,10]$ demonstrated that the presence of free surface can significantly change the phase transformation process of NiTi SMA at the nano scale. Therefore, it can be expected that the introduction of free surfaces through spherical pores will produce similar effects on the phase transformation process of NiTi SMA at the nano scale.

\subsection{Shape memory effect (SME)}

Figure 2 shows the evolution of different phases i.e. B2, B19 and B19' in non-porous and porous NiTi, at different temperatures. More details about the structural characterizing are provided in the supplementary material. For the non-porous NiTi simulation cell (Fig. 2 (a1)(d1)), evolution of different phases with temperature shows a homogeneous texture, while, in 
the case of the porous NiTi simulation cell (Fig. 2 (a2)-(d2)), evolution of different phases shows an inhomogeneous texture, specifically near the pore surface. In particular, the results show that two variants of B19 phase are stabilized near the pore surface of the porous NiTi simulation cell, but not observed for the case of the non- porous NiTi simulation cell. Similar observations are also reported in other studies [4-6,37], where due to the presence of internal/ free surfaces, B19 variants are stabilized, which are not typical of bulk nonporous NiTi. Thus, in non-porous $\mathrm{NiTi}$, the phase transformation process/ path is $\mathrm{B} 2 \rightarrow \mathrm{B} 19^{\prime}$ (or vice versa); however for porous NiTi it becomes B2 $\rightarrow$ B19 $\rightarrow$ B19' (or vice versa).

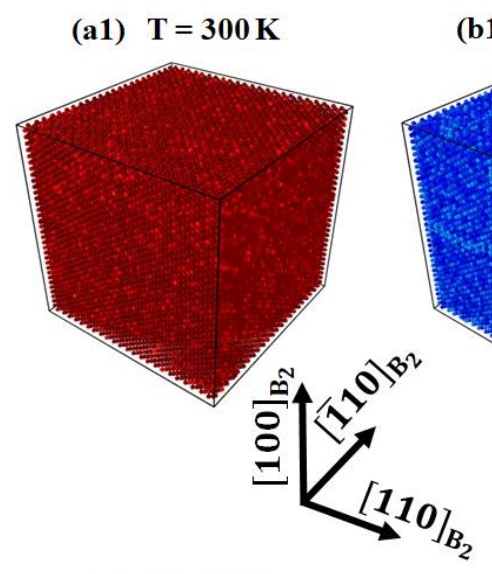

(a2) $\mathrm{T}=300 \mathrm{~K}$

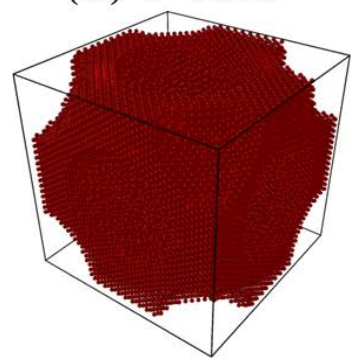

(b1) $\mathrm{T}=350 \mathrm{~K}$

(b2) $\mathrm{T}=350 \mathrm{~K}$

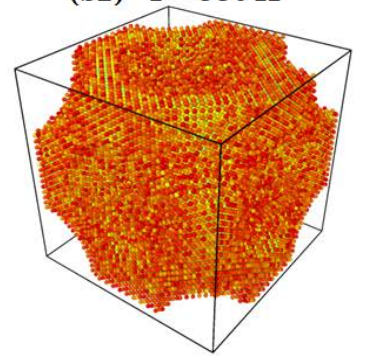

(c1) $\mathrm{T}=\mathbf{4 0 0} \mathrm{K}$

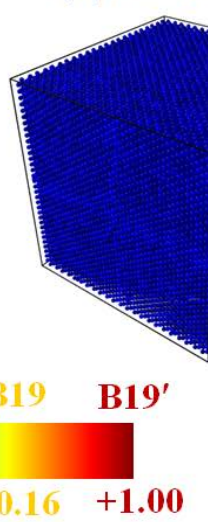

(c2) $\mathrm{T}=400 \mathrm{~K}$

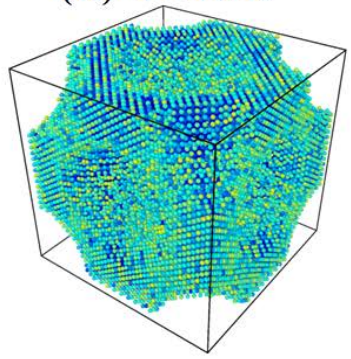

(d1) $\mathrm{T}=450 \mathrm{~K}$

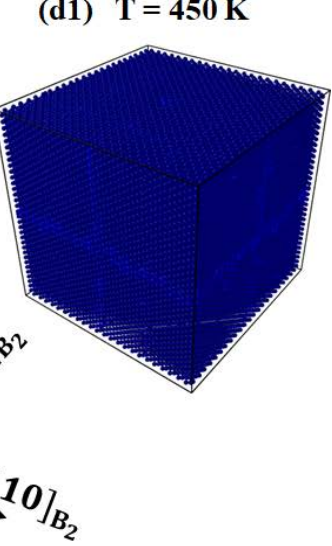

(d2) $\mathrm{T}=450 \mathrm{~K}$

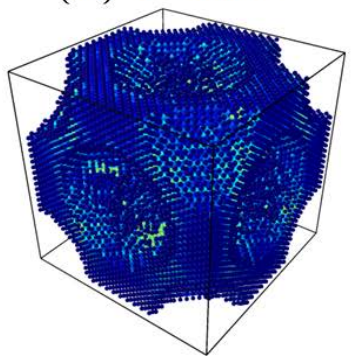

Figure-2: Evolution of different phases (B2, B19, and B19') at different temperatures (T = 300 K, $350 \mathrm{~K}, 400 \mathrm{~K}$ and $450 \mathrm{~K}$ ) for non-porous NiTi ((a1) - (d1)) and $40 \%$ porous ((a2) - (d2)) NiTi. Crystallographic orientations are also shown in this figure.

To demonstrate the above statement, Fig. 3 is provided, which shows the zoomed-in view of the microstructure of porous NiTi in the vicinity of a pore as a function of temperature. The pore surface is marked with a white broken line. Here, Fig. 3a shows the unit cells of the orthorhombic B19 phase, at $\mathrm{T}=400 \mathrm{~K}$. Figures 3 (b)-(d) show the evolution of two different variants of the B19 phase (near the pore surface) at different temperatures, indicating that the evolution of the B19 phase (on or near the pore surface) in porous NiTi happens at the expense of the B19' phase. A similar observation is also reported in a recent MD study on free-standing NiTi nanoparticles [5]. As reported in that study [5], thermally induced martensitic phase transformation (evolution of B19' phase from B2 phase) process can be suppressed due to the formation of near-parent-phase (similar to B2 phase) structure on the nanoparticle surface. 


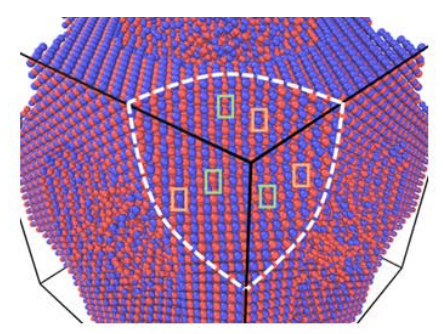

(b) $\mathbf{T}=350 \mathrm{~K}$

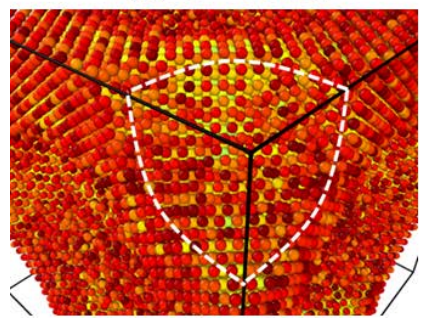

(a)

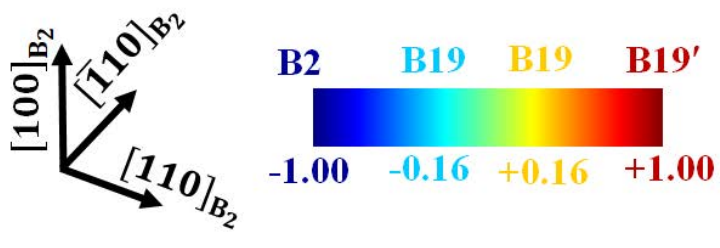

(c) $\mathrm{T}=400 \mathrm{~K}$

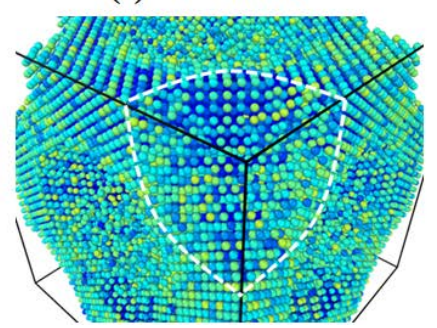

(d) $\mathbf{T}=450 \mathrm{~K}$

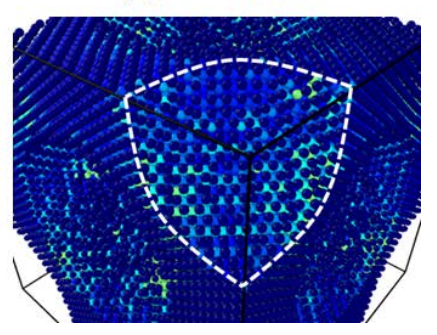

Figure-3: Zoomed-in view showing the evolution of different variants of B19 phase near the pore surface of porous NiTi; (a) shows the unit cell of B19 phase at T $=400 \mathrm{~K}$ and (b)-(d) show the evolution of different variants of B19 phase at T $=350 \mathrm{~K}, 400 \mathrm{~K}$ and $450 \mathrm{~K}$.

Figure 4 shows the effect of porosity on the thermally induced martensite phase fraction evolution. Specifically, Fig. 4 (a) and (b) show the evolution of martensite phase fraction, as a function of porosity for the martensitic and austenitic phase transformation process, respectively. It can be observed that for the entire temperature range, with increasing porosity the martensite phase fraction value increases and the phase transformation process starts at a higher temperature, i.e., earlier as compared to that in non-porous NiTi. Fig. 4 (c) shows the width of the thermal hysteresis between the austenitic and martensitic phase transformation process. Interestingly, with increasing porosity, the width of the thermal hysteresis decreases and the width is maximum for non-porous NiTi.

The evolution of martensite phase fraction is found to follow the Richards equation [39], i.e., Eqns. (1), with respect to temperature [7,9,10,34,39], where the parameters entering the equation depend on the porosity. Eqn. (1.1) describes martensitic, and Eqn. (1.2) austenitic phase transformation process, i.e.

$$
\begin{aligned}
& \xi_{A M}(T, p)=1-\left[1+\exp \left\{-\beta(p)\left(\frac{T-M_{m}(p)}{M_{S}(p)-M_{f}(p)}\right)\right\}\right]^{\{-1 / v(p)\}} \\
& \xi_{M A}(T, p)=1-\left[1+\exp \left\{-\beta(p)\left(\frac{T-A_{m}(p)}{A_{f}(p)-A_{s}(p)}\right)\right\}\right]^{\{-1 / v(p)\}}
\end{aligned}
$$

where, $\beta$ and $v$ are model parameters controlling the shape of the evolution kinetics curves, $A_{s}$ and $A_{f}$ are the austenitic start and finish temperature, respectively, $M_{s}$ and $M_{f}$ are the martensitic start and finish temperature, respectively, $A_{m}$ and $M_{m}$ are the maximum rate of the austenitic and martensitic phase transformation temperature, respectively. Here, $A_{m}$ and $M_{m}$ represent the temperatures at which the conversion rate of austenite and martensite phase to other phases are maximum; i.e. at $A_{m}$ and $M_{m}$ temperatures, $\left|d \xi_{M A} / d T\right|$ and $\left|d \xi_{A M} / d T\right|$ are maximum, respectively. From Eq. 1.1 and 1.2 it is possible to estimate these absolute maximum values of the austenitic and martensitic phase transformation rate as 


$$
\begin{aligned}
& \left|\frac{d \xi_{A M}\left(T=M_{m}(p), p\right)}{d T}\right|=\left(\frac{1}{M_{S}(p)-M_{f}(p)}\right)\left(\frac{\beta(p)}{v(p)}\right) \\
& \left|\frac{d \xi_{M A}\left(T=A_{m}(p), p\right)}{d T}\right|=\left(\frac{1}{A_{f}(p)-A_{S}(p)}\right)\left(\frac{\beta(p)}{v(p)}\right)
\end{aligned}
$$

A fitting process shows that both of these model parameters (i.e. $\beta$ and $v$ ) decrease with increasing porosity, while the phase transformation temperatures increases with increasing porosity. Here, those two model parameters mainly control the shape of the martensite phase fraction evolution curves. Both model parameters decrease linearly with increasing porosity, as is evident from Table-S1 in the supplementary material.
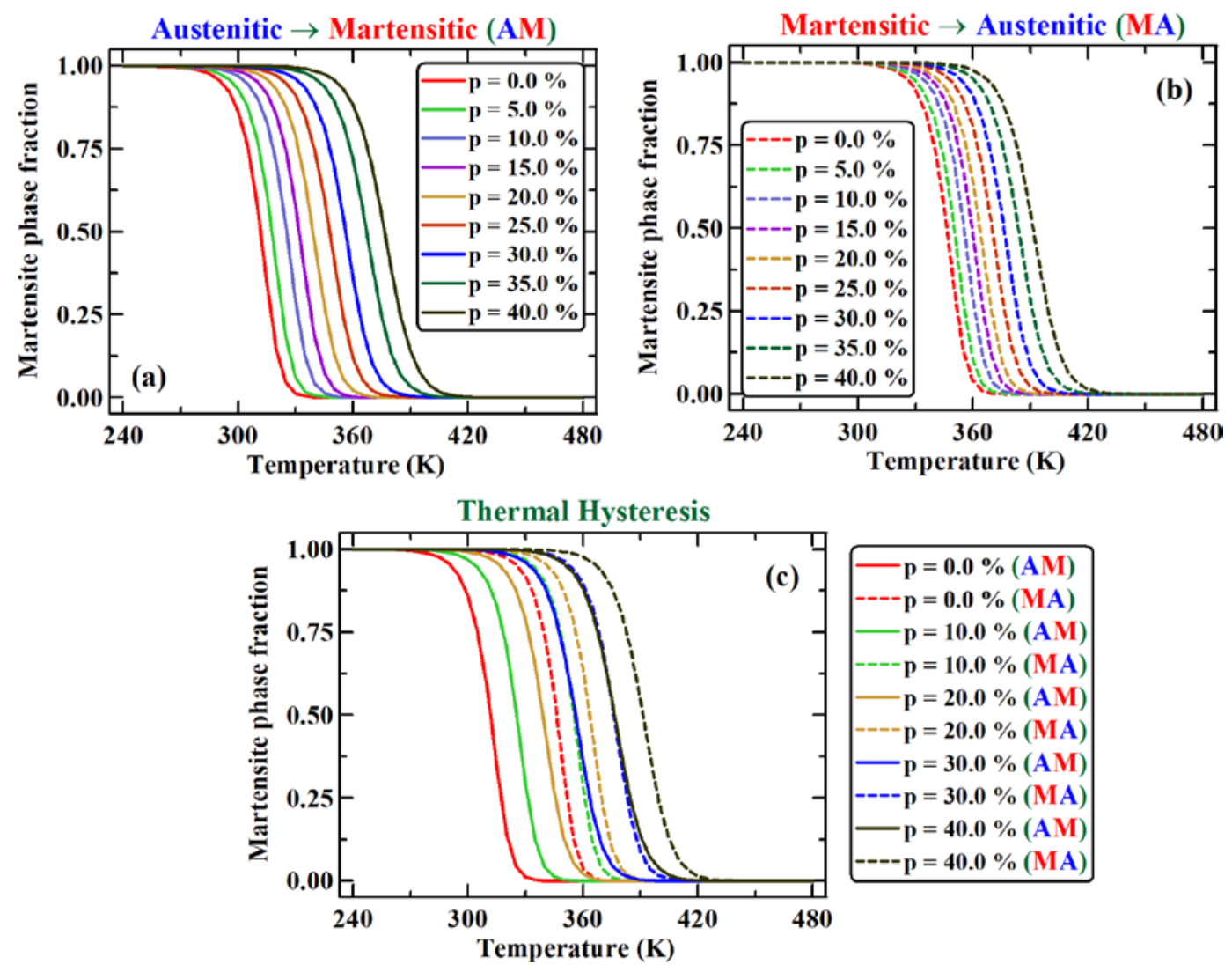

Figure-4: Variation of the martensite phase fraction with respect to temperature for various levels of porosity. (a) Martensitic phase transformation process. (b) Austenitic phase transformation process. (c) Variation of thermal hysteresis width between austenitic and martensitic phase transformation process for various levels of porosity.

Figure 5 shows the effects of porosity on the phase transformation temperatures and the width of the thermal hysteresis loop. Fig. 5 (a) and (b) respectively show the variation of martensitic (i.e. Fig. 5 (a) martensite start $\left(M_{s}\right)$, martensite finish $\left(M_{f}\right)$, and the maximum rate of martensitic transformation $\left(M_{m}\right)$ ) and austenitic (i.e. Fig. 5 (b) austenite start $\left(A_{s}\right)$, austenite finish $\left(A_{f}\right)$, and the maximum rate of austenitic transformation $\left(A_{m}\right)$ ) phase transformation temperatures with respect to porosity. 


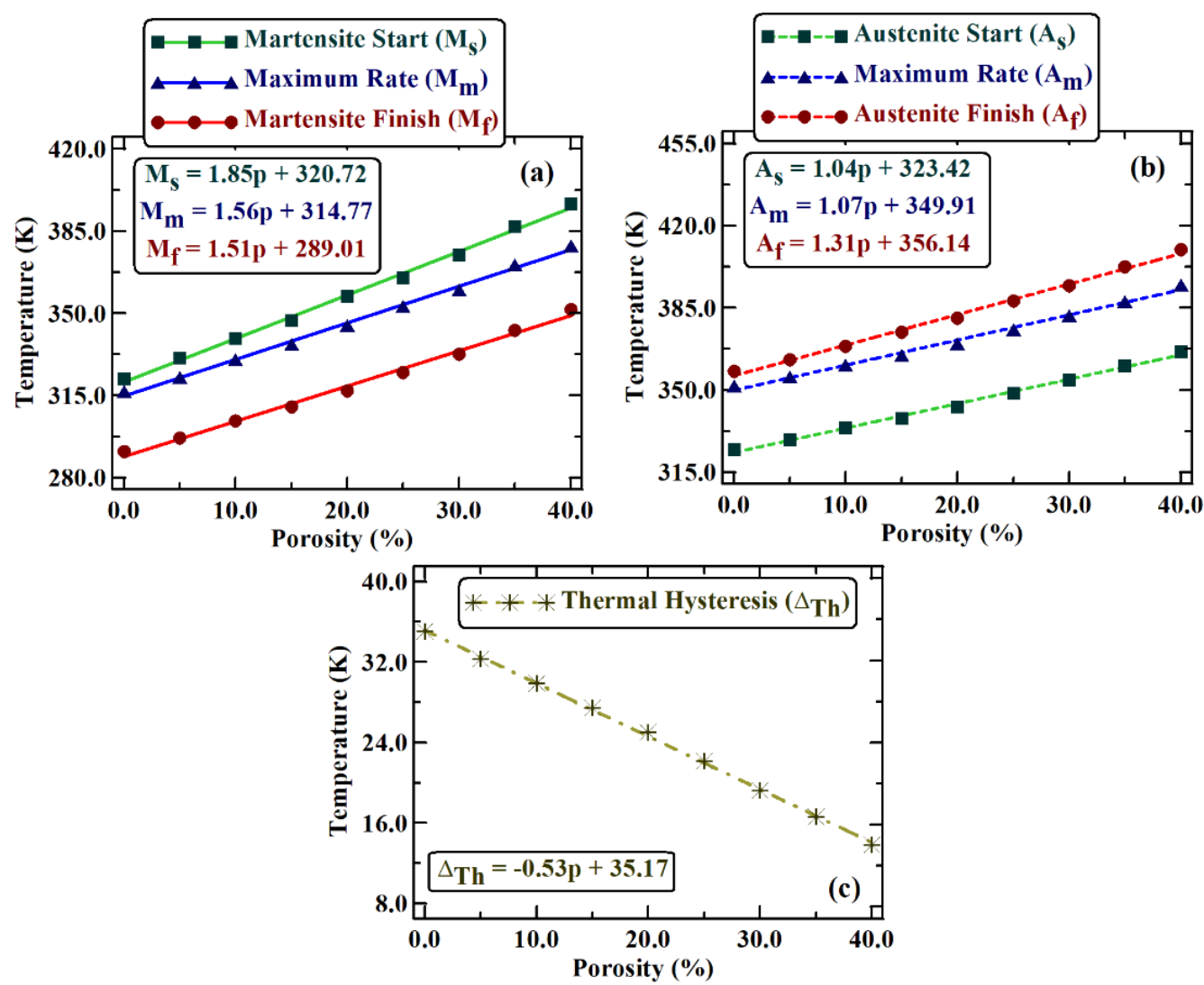

Figure-5: Variation of the transformation temperatures with respect to the porosity of NiTi (a) martensitic phase transformation process, (b) austenitic phase transformation process, and (c) the width of thermal hysteresis, due to the phase transformation process.

The temperatures at which the respective phase transformations are initiated and completed increase with porosity and interestingly show a linear variation within the adopted range of porosity. Specifically, with increasing porosity, there is a corresponding increase in available surface per unit volume, thereby hastening the phase transformation, and leading to higher phase transformation temperatures. A critical difference is the presence of the metastable B19 phase in porous NiTi, while the B19' phase is characteristic of non-porous NiTi. In addition, the width of the thermal hysteresis corresponding to a complete cycle of phase transformation as a function of temperature (i.e. the martensitic followed by the austenitic phase transformation) decreases linearly with increasing porosity, which once again correlates to the role of the pore surface in hastening the respective phase transformation process.

\subsection{Super-elasticity (SE)}

Based on the results described in the previous section, it is expected that due to the change in martensite phase fraction evolution and transformation temperatures with porosity, the stressstrain response of porous NiTi will also differ from that of non-porous one. In this regard, we initially discuss the role of stress on the super-elasticity of porous vs non-porous NiTi systems at a fixed temperature equaling $400 \mathrm{~K}$. Note that the dependence of the superelastic behavior as a function of temperature is discussed later in this section. 
Figure 6 shows the stress strain response (at $T=400 \mathrm{~K}$ ) and the respective evolution of the different phases i.e. B2, B19 and B19' (due to applied stress), for the non-porous NiTi (Fig. 6 (a)) and porous NiTi systems (Fig. 6 (b), for $40 \%$ porosity).
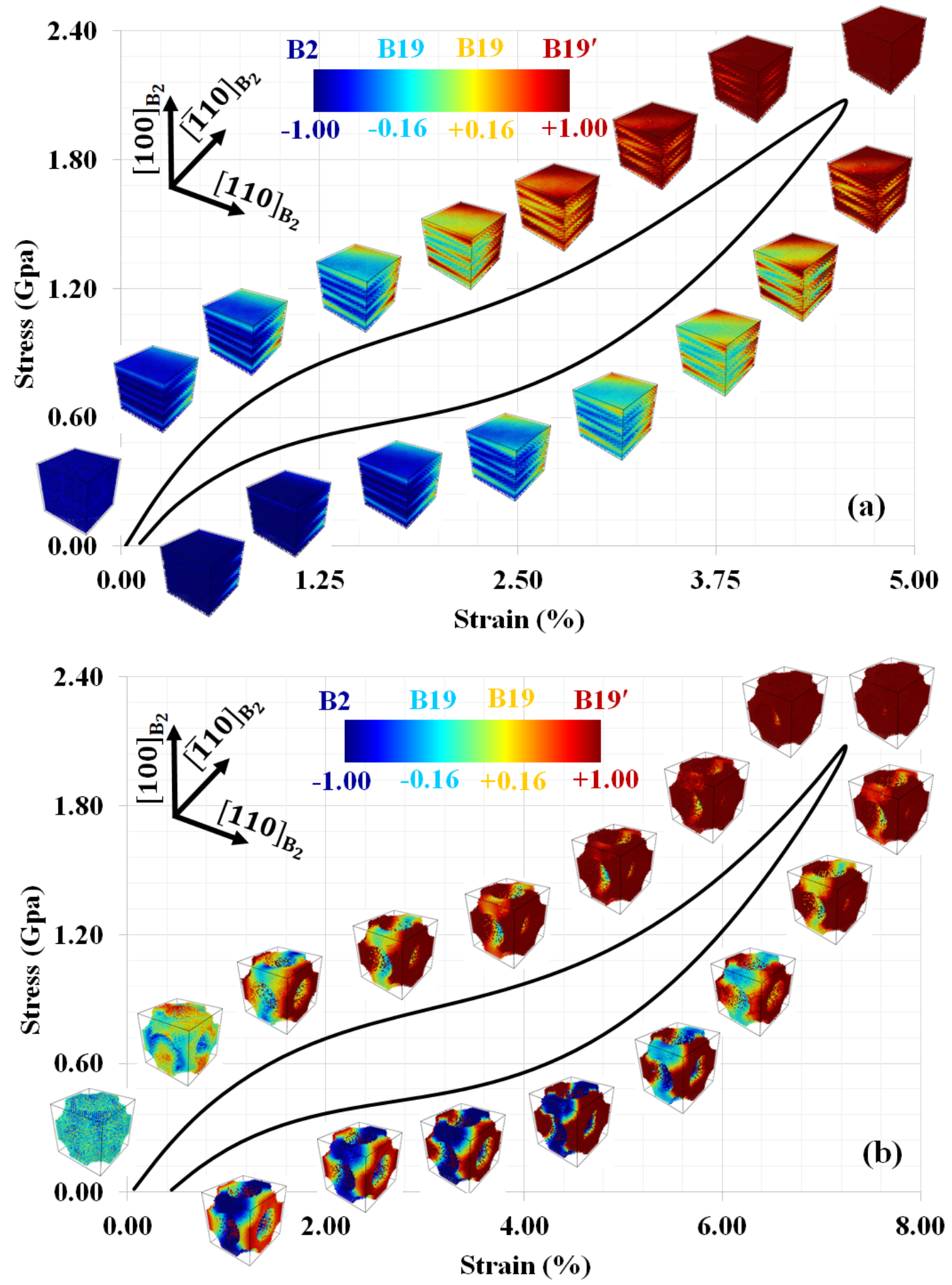

Figure-6: Stress strain response and evolution of different phases (B2, B19, and B19') at T = $400 \mathrm{~K}$ temperature for (a) non-porous NiTi and (b) porous NiTi with porosity at $40 \%$.

Here, for both non-porous and porous NiTi, the transformation follows the following path: B2 $\rightarrow$ B19 $\rightarrow$ B19' (or vice versa). However, in non-porous NiTi, different phases grow in a layer- 
by-layer fashion, whereas, for porous $\mathrm{NiTi}$, the phase transformation is initiated on the pore surfaces, pointing to differences in phase transformation initiation for non-porous and porous NiTi. Further details about the evolution of different phases under stress and stress induces phase transformation process (SE) in nonporous and porous NiTi are provided subsequently.

Figure 7 show the evolution of different phases in the nonporous NiTi simulation cell under applied stress, and a temperature equaling $400 \mathrm{~K}$. Figures 7 (a)-(d) show the evolution of different phases under loading for a stress cycle $\left(\sigma_{l}=\right.$ 0.7 GPa, 1.1 GPa, 1.5 GPa, and 1.9 GPa). Similarly, Figs. 7 (e)-(h) show the evolution of phases under stress unloading (i.e., at $\sigma_{u l}=1.3 \mathrm{GPa}, 0.6 \mathrm{GPa}, 0.3 \mathrm{GPa}$, and $0.1 \mathrm{GPa}$ ). During the loading stage, the B2 phase first transforms into the metastable B19 phase which then transforms into stable B19' phase, while the reverse sequence characterizes the unloading process. As a result, for a complete loading-unloading cycle at $400 \mathrm{~K}$, there is negligible residual strain. Interestingly, during both loading and unloading, the respective transformations occur along the $[100]_{\mathrm{B}_{2}}$ direction.
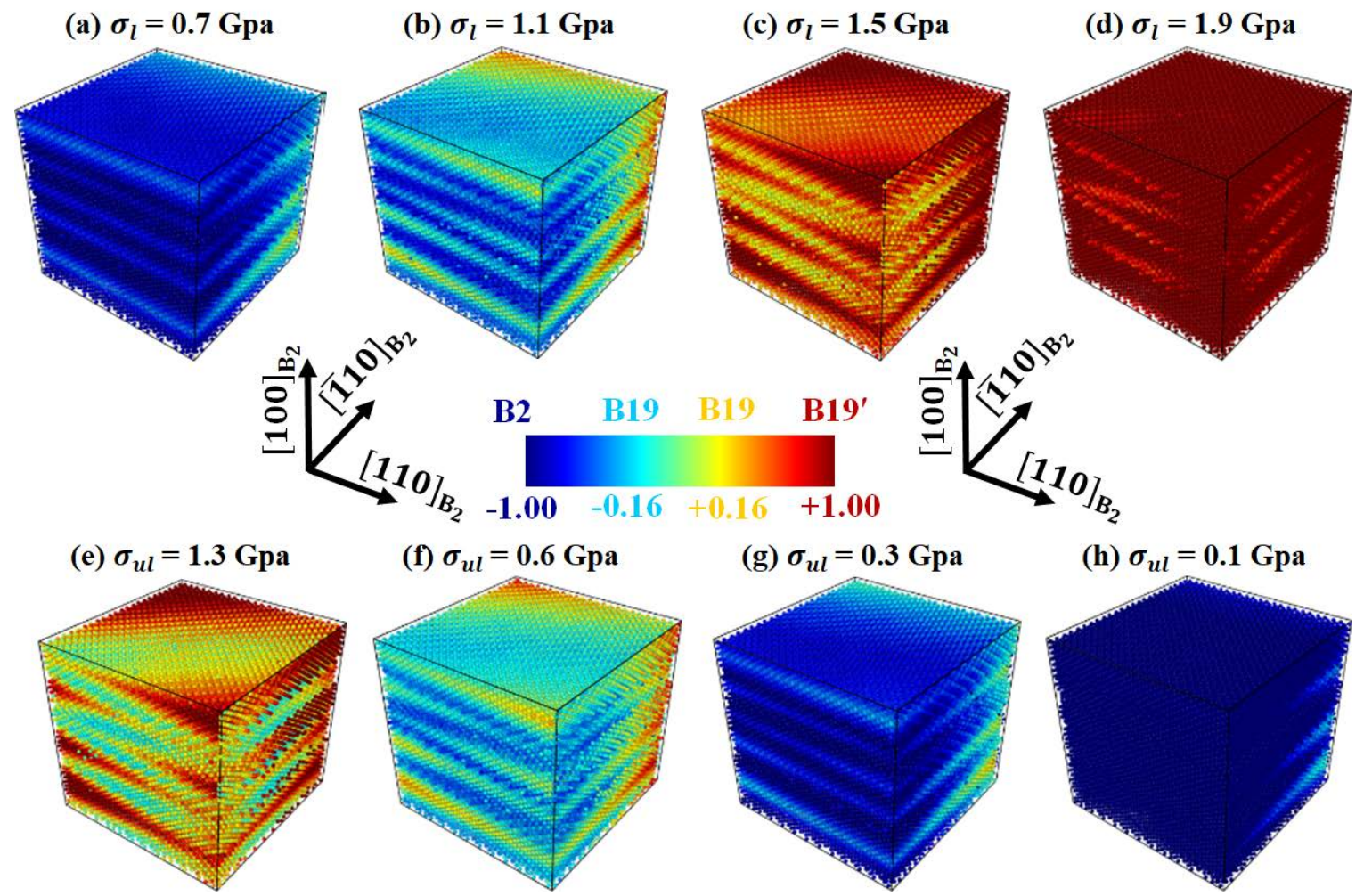

Figure-7: Evolution of different phases (B2, B19, B19') in nonporous NiTi simulation cell at $\mathrm{T}=400 \mathrm{~K}$, under different stress level, for a loading and unloading cycle. For (a)-(d) under loading cycle with stress level $\sigma_{l}=0.7 \mathrm{GPa}, 1.1 \mathrm{GPa}, 1.5 \mathrm{GPa}$, and $1.9 \mathrm{GPa}$; and for the (e)-(h) under loading cycle with stress level $\sigma_{u l}=1.3 \mathrm{GPa}, 0.6 \mathrm{GPa}, 0.3 \mathrm{GPa}$, and $0.1 \mathrm{GPa}$.

Figure 8 shows the evolution of different phases in porous NiTi under the same stress level as in nonporous NiTi) during loading (Fig. 8 (a)-(d)) and unloading (Fig. 8 (e)-(h)), at a temperature equaling $400 \mathrm{~K}$. Unlike non-porous NiTi, in porous NiTi the phase transformation 
of B2 occurs along the $[110]_{\mathrm{B}_{2}}$ direction. More importantly, the phase transformation results in two variants of B19 phases in addition to the B19' phase, similar to previous MD based observations that examined the role of free surfaces on the stabilization of the two B19 variants [6, 37]; for comparison, the nonporous system is characterized by the presence of only one metastable B19 phase. Another significant difference between the porous and nonporous systems is the residual strain present in the parent B2 phase, after the porous system has been subjected to a complete loading-unloading cycle. This is a direct consequence of the presence of B19 and B19' remnants within the parent B2 porous system even at zero stress as discussed later in this section.

(a) $\sigma_{l}=0.7 \mathrm{Gpa}$

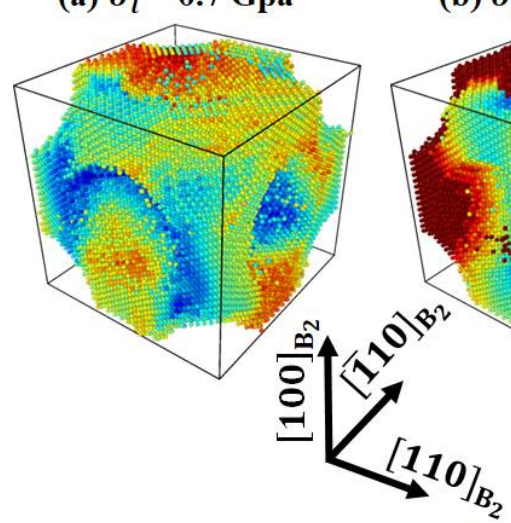

(e) $\sigma_{u l}=1.3 \mathrm{Gpa}$

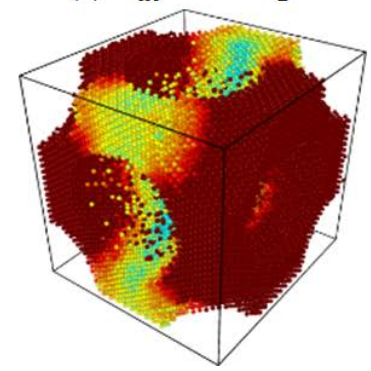

(b) $\sigma_{l}=1.1 \mathrm{Gpa}$

(f) $\sigma_{u l}=0.6 \mathrm{Gpa}$

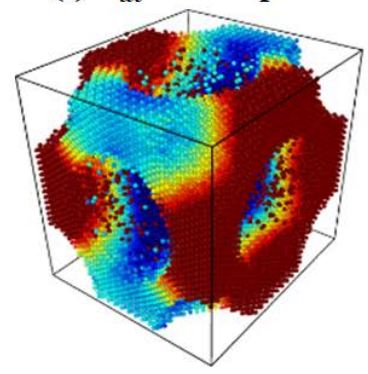

(c) $\sigma_{l}=1.5 \mathrm{Gpa}$

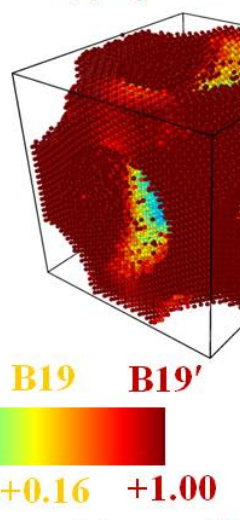

(g) $\sigma_{u l}=0.3 \mathrm{Gpa}$

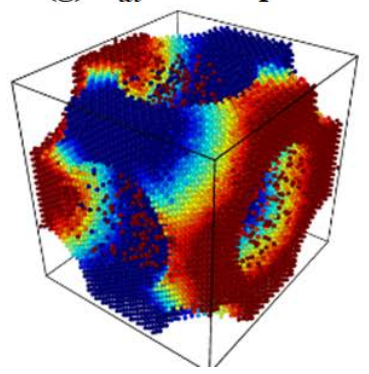

(d) $\sigma_{l}=1.9 \mathrm{Gpa}$

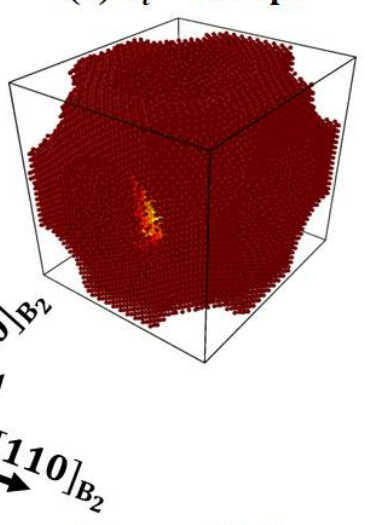

(h) $\sigma_{u l}=0.1 \mathrm{Gpa}$

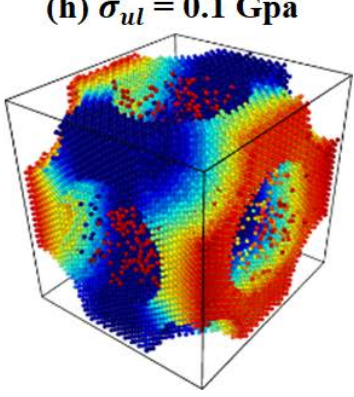

Figure-8: Evolution of different phases (B2, B19, B19') in porous NiTi simulation cell (40 \% porosity) at $\mathrm{T}=400 \mathrm{~K}$, under different stress levels, during a loading and unloading cycle. For (a)-(d) for loading with stress level $\sigma_{l}=0.7 \mathrm{GPa}, 1.1 \mathrm{GPa}, 1.5 \mathrm{GPa}$, and 1.9 GPa; and for (e)(h) for unloading with stress level $\sigma_{u l}=1.3 \mathrm{GPa}, 0.6 \mathrm{GPa}, 0.3 \mathrm{GPa}$, and $0.1 \mathrm{GPa}$.

On a related note, we did not observe the formation of the body-centered orthorhombic (BCO) phase during loading or unloading. This is attributed to the relative low tensile-strains attained in our MD simulations as compared to Zhong et al. [37], or Mirzaeifar et al [6].

Figure 9 shows the stress-strain response of NiTi for various levels of porosity as a function of temperature. It can be seen that for a given porosity, increasing temperature leads to a consequent increase in the stress-strain loop size and critical transformation stress. Other studies on the non-porous and porous NiTi SMA (based on simulations at either atomic or meso scale and continuum scale), report such similar increase in transformation stress with increasing temperature [6,12-15]. This observation is directly attributable to the increased amount of austenite at higher temperatures, which leads to (i) higher critical stress to complete the phase 
transformation process and (ii) increase in the area under the respective stress-strain loops with increasing temperature.
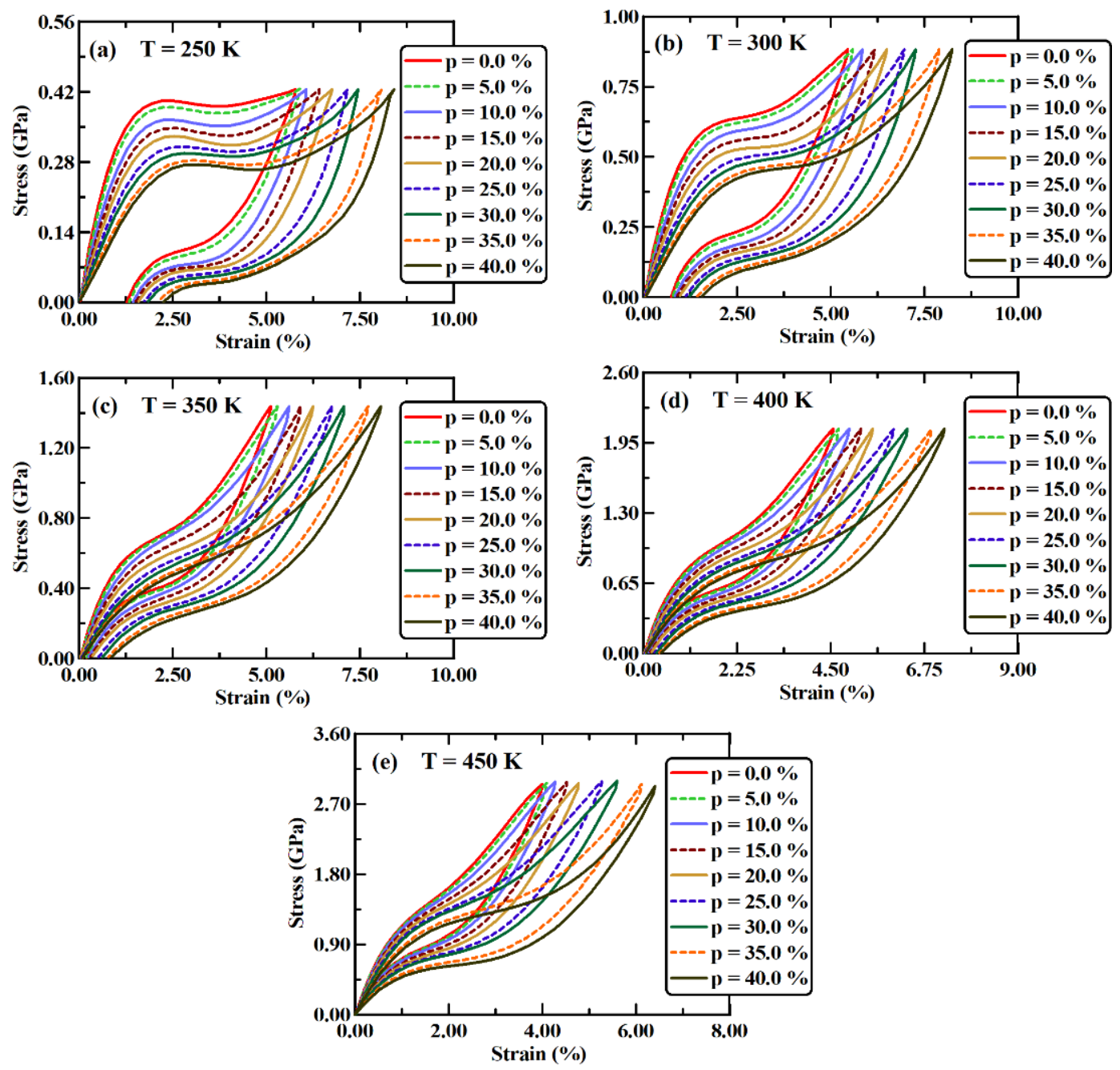

Figure-9: Stress strain response of non-porous and porous $\mathrm{NiTi}$ (with various levels of porosity) at various temperature (a) $\mathrm{T}=250 \mathrm{~K}$, (b) $\mathrm{T}=300 \mathrm{~K}$, (c) $\mathrm{T}=350 \mathrm{~K}$, (d) $\mathrm{T}=400 \mathrm{~K}$ and (e) $\mathrm{T}=450 \mathrm{~K}$.

Consistent with other macroscale studies [12,14,16,18,19,22], with increasing porosity the critical phase transformation (PT) stress decreases, and the stress-strain loop size increases. Further, for a given temperature, with increasing porosity, the amount of the martensitic fraction increases and the critical transformation stress decreases. The increase in the stressstrain loop size can be understood in terms of the transformation path namely B2 $\rightarrow$ B19 $\rightarrow$ B19'. Specifically, as pointed earlier, for the non-porous system the intermediate B19 phase is not observed and the B2 phase directly transforms to the B19' phase, while with increasing porosity, the intermediate B19 phase is stabilized before ultimately transforming into the B19' phase. This correlates to a substantial increase in the maximum strain required to achieve B19 $\rightarrow$ B19' transformation as porosity increases, leading to a consequent increase in the stressstrain loop size.

Figure 10 shows the variation of the phase transformation (PT) stress (initiation and completion) for the forward and reverse transformation process for various porosities and 
temperatures. In this context, it is worth noting that in the MD simulations conducted in this study, 3-D periodic boundary conditions were used, while surface-effects and grain boundaries were not explicitly considered. Thus the obtained PT stress-values represent an upper limit as compared to continuum scale based experimental/computational results where surface and grain boundary effects can serve as additional constraints.

Similar to the experimental results reported in [15], our MD simulation results shows that with increasing temperature, the critical values of forward and reverse PT stress increase. Interestingly, with increasing porosity, the critical values of forward and reverse PT stress decrease linearly, as reported in the several continuum scale studies [12-14,16-19,27,40]. The values of this linear model parameters (i.e. $\beta_{s}^{A M}, \beta_{f}^{A M}, \beta_{s}^{M A}, \beta_{f}^{M A}$; and $\alpha_{s}^{A M}, \alpha_{f}^{A M}, \alpha_{s}^{M A}, \alpha_{f}^{M A}$ ) are provided in Table-S2 in the supplementary material, where, with increasing temperature, $\beta$ value increases and $\alpha$ value decreases.
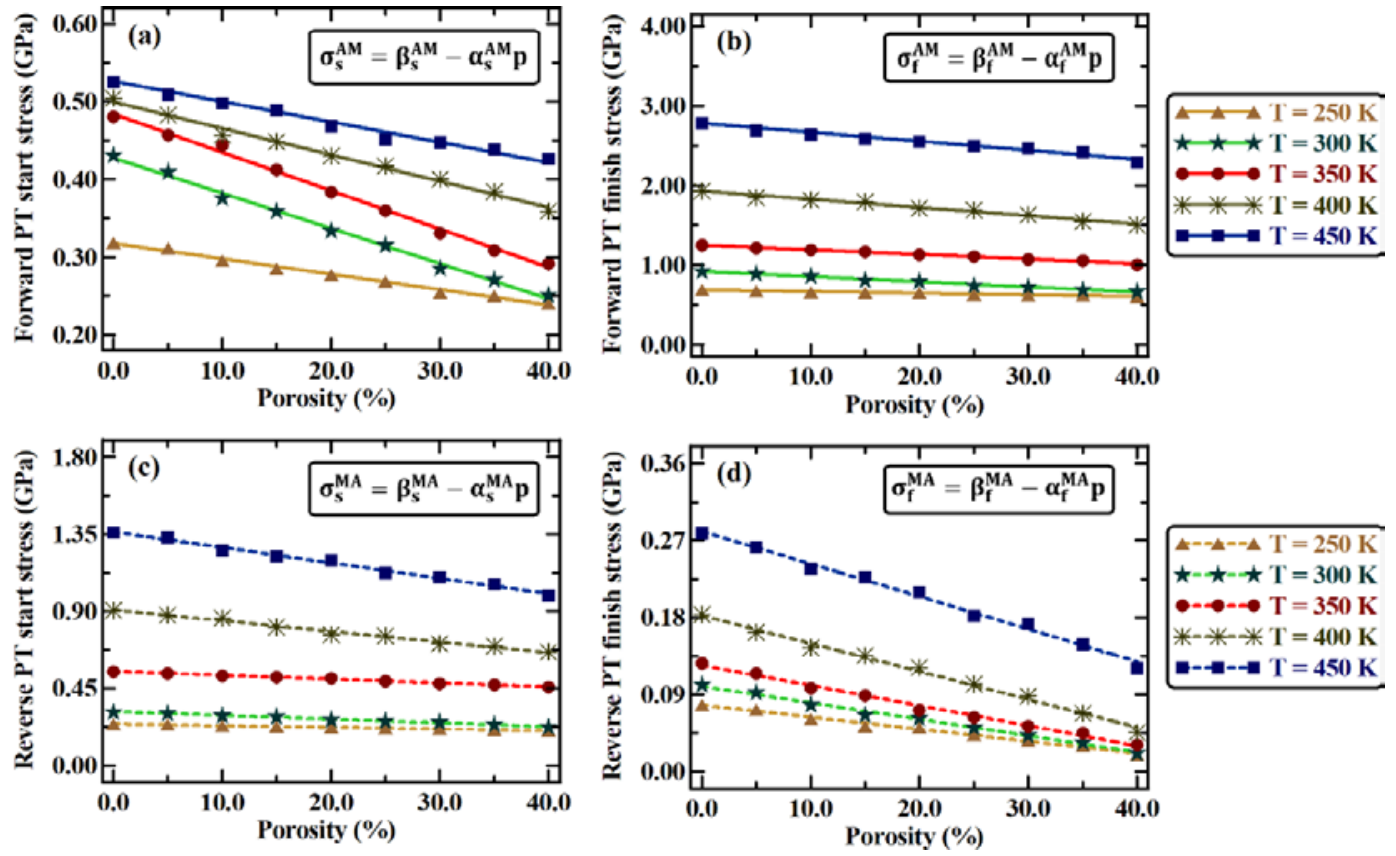

Figure-10: Variation of phase transformation (PT) stress with respect to porosity for various temperatures (a) forward PT start stress $\left(\sigma_{s}^{A M}\right)$, (b) forward PT finish stress $\left(\sigma_{f}^{A M}\right)$, (c) reverse PT start stress $\left(\sigma_{s}^{M A}\right)$, (d) reverse PT finish stress $\left(\sigma_{f}^{M A}\right)$.

Another consequence of the formation of the intermediate B19 phase is the presence of residual strain especially in porous NiTi after the completion of a loading-unloading cycle. Details about the energy dissipation capacity and residual strain of non-porous and porous NiTi as a function of temperature and porosity are shown in Figure 11. Fig. 11 (a) and (b) show the variation of the residual strain and PT induced strain with respect to porosity and for various temperatures. At any constant temperature, with increasing porosity, both the residual strain and the PT induced strain increase rapidly, and the increase can be fitted to an exponential function. Different coefficients of this exponential model are provided in Table-S3 in the supplementary material, where it is shown that with increasing temperature, $\beta_{r}$ or $\beta_{l}$ values decrease and $\alpha_{r}$ or $\alpha_{l}$ values increase. 

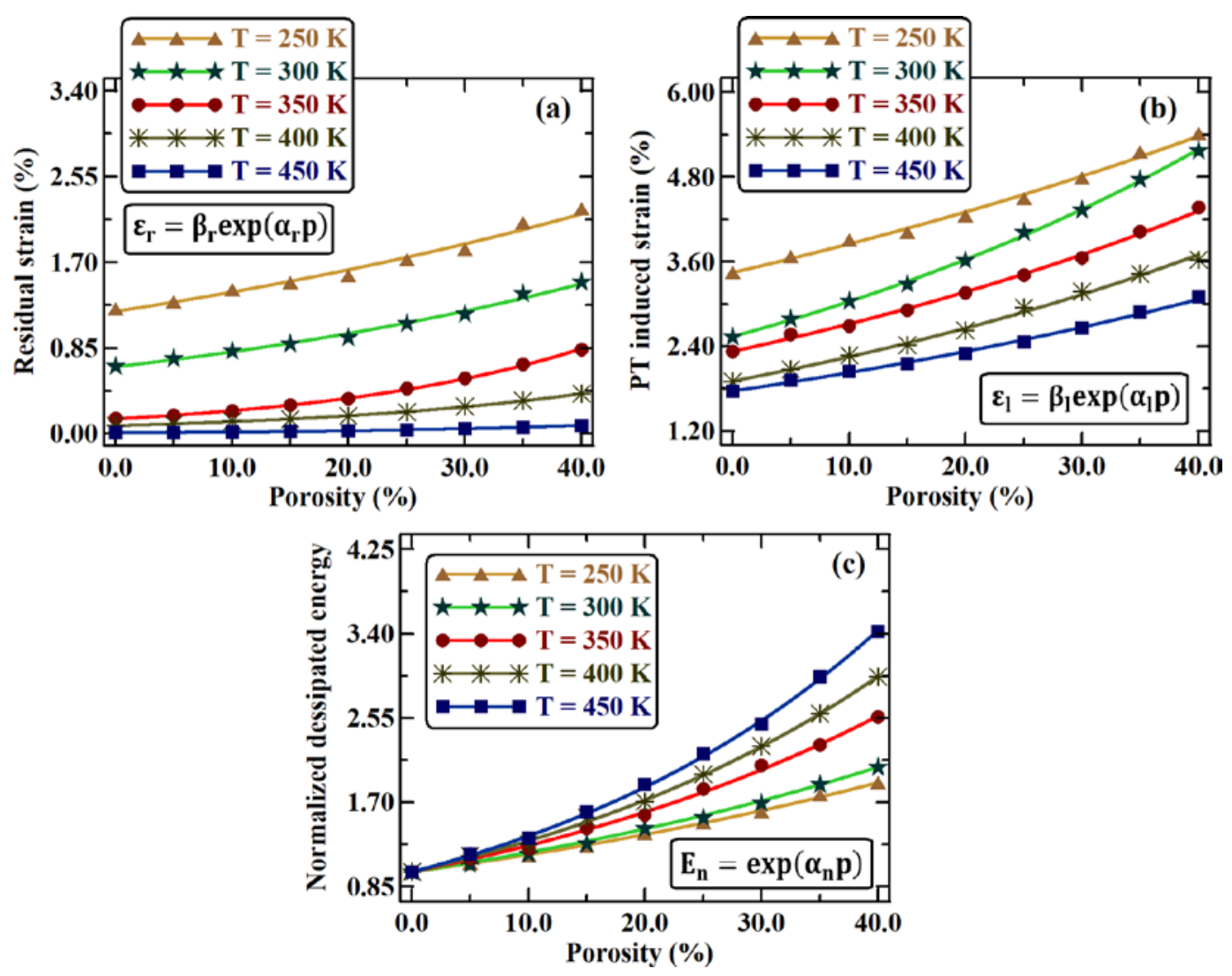

Figure-11: Effect of porosity and temperature on different parameters of the stress strain response curves (a) residual strain, (b) maximum strain and (c) normalized hysteresis energy dissipation capacity. Energy dissipation capacity of porous NiTi is normalized with respect to the energy dissipation capacity of non-porous NiTi.

At low temperatures, for both non-porous and porous NiTi, the residual strain is very high. This is especially evident for temperatures below the martensite start temperature $M_{s}$, where under loading, there is inter-conversion between B19 and B19' variants; upon unloading, there is incomplete recovery, leading to significant amount of residual strain. However at temperatures above $M_{s}$, there is an increase in the amount of the B2 phase with increasing temperature and with increasing B2 phase fraction, the ability to form the intermediate martensitic phase decreases leading to a decrease in residual strain. Fig. 11 (c) shows the variation of normalized (per unit mass) energy dissipation capacity of porous NiTi at various temperatures. It increases exponentially with increasing porosity, and the values of the exponential model parameters are provided in Table-S3 in the supplementary material, where it is shown that with increasing temperature, $\alpha_{n}$ values increase. Here, for a given temperature, the energy dissipation capacity of porous $\mathrm{NiTi}$ is normalized with respect to the energy dissipation capacity of non-porous NiTi, which in turn is related to the size of the stress-strain loop. The energy dissipation capacity increases with (i) increasing porosity at a given temperature, and (ii) increasing temperature for a given porosity which is correlated to a larger extent of austenite to martensite phase transformation [41].

\section{Conclusions}


In summary, the present study shows the effects of porosity on the shape memory effect and superelasticity of NiTi SMA. To this end, classical MD simulations are performed on nonporous and porous NiTi, employing an appropriate EAM interatomic potential to examine the interplay between temperature and stress on shape memory and superelasticity properties. MD simulation results shows that with increasing porosity (which increases the internal effective surface area), phase transformation temperature increases linearly and thermal hysteresis loop size decreases linearly. At a given temperature, increasing porosity leads to an increase in the martensite phase fraction, which in turn underlies the stress induced phase transformation process and the stress-strain response i.e., superelasticity (SE) of NiTi SMA. At a constant temperature, with increasing porosity, the critical stress of phase transformation decreases and the NiTi SMA experiences a higher level of strain at the same level of stress. Further, compared to non-porous $\mathrm{NiTi}$, the residual strain and the energy dissipation capacity of porous NiTi increases with increasing porosity. Also, the effect of temperature on superelasticity of nonporous and porous NiTi are studied, showing that with increasing temperature the critical stress for phase transformation and the hysteretic energy dissipation capacity increase, and the residual strain decreases. Taken together, incorporation of porosity at nanoscale significantly alters the microstructure evolution process in NiTi (compared to non-porous $\mathrm{NiTi}$ ) and therefore changes the shape memory effect (SME) and superelasticity (SE) properties of porous NiTi. As porosity alters the SME and SE properties of porous NiTi compared to that of nonporous $\mathrm{NiTi}$, results from this study can be used to develop nano-scale tunable devices and dampers. For example, adopting different geometries for NiTi samples in conjunction with grading in porosity, functionally graded non-porous NiTi samples can be created, which can provide different SME and SE properties depending on the grading. Such functionally graded non-porous NiTi can be used to develop tunable devices and dampers at the nano-scale.

\section{Acknowledgements}

The reported exploratory work became possible through the support of SG by the University of Arizona. The authors would like to acknowledge Prof. Ting Zhu (Woodruff school of mechanical engineering at Georgia Institute of Technology) for providing the interatomic potential parameters.

\section{References}

[1] T. Tadaki, K. Otsuka, K. Shimizu, Annual Review of Materials Science 18 (1988) 2545.

[2] Jan Van Humbeeck, Materials Science and Engineering: A 273-275 (1999) 134-148.

[3] T. Duerig, A. Pelton, D. Stockel, Materials Science and Engineering: A 273-275 (1999) 149-160.

[4] D. Mutter, P. Nielaba, Journal of Alloys and Compounds 577S (2013) S83-S87.

[5] Z. Zhang, X. Ding, J. Deng, J. Cui, J. Sun, T. Suzuki, K. Otsuka, X. Ren, The Journal of Physical Chemistry C 117 (2013) 7895-7901. 
[6] R. Mirzaeifar, K. Gall, T. Zhu, A. Yavari, R. Desroches, Journal of Applied Physics 115 (2014) 194307.

[7] S. Gur, G.N. Frantziskonis, Smart Materials Research 2016 (2016) 7512642.

[8] D. Mutter, P. Nielaba, European Physical Journal B 84 (2011) 109-113.

[9] S. Gur, G.N. Frantziskonis, Modelling and Simulation in Materials Science and Engineering Modelling 24 (2016) 075006.

[10] G.N. Frantziskonis, S. Gur, Modelling and Simulation in Materials Science and Engineering 25 (2017) 045002.

[11] B.T. Lester, T. Baxevanis, Y. Chemisky, D.C. Lagoudas, Acta Mechanica 226 (2015) 3907-3960.

[12] M.A. Qidwai, P.B. Entchev, D.C. Lagoudas, V.G. DeGiorgi, International Journal of Solids and Structures 38 (2001) 8653-8671.

[13] P.B. Entchev, D.C. Lagoudas, Mechanics of Materials 34 (2002) 1-24.

[14] P.B. Entchev, D.C. Lagoudas, Mechanics of Materials 36 (2004) 893-913.

[15] S. Nemat-Nasser, Y. Su, W.G. Guo, J. Isaacs, Journal of the Mechanics and Physics of Solids 53 (2005) 2320-2346.

[16] Y. Zhao, M. Taya, Journal of Applied Mechanics 74 (2007) 291.

[17] Y. Zhu, G. Dui, Acta Mechanica Solida Sinica 24 (2011) 289-298.

[18] M. Panico, L.C. Brinson, International Journal of Solids and Structures 45 (2008) 56135626.

[19] B.S. Shariat, Y. Liu, G. Rio, Journal of Intelligent Material Systems and Structures 25 (2014) 1445-1455.

[20] P. Zhu, A.P. Stebner, L. Catherine Brinson, Smart Materials and Structures 23 (2014) 104008.

[21] V. Sepe, F. Auricchio, S. Marfia, E. Sacco, Smart Materials and Structures 24 (2015) 085035.

[22] M.J. Ashrafi, J. Arghavani, R. Naghdabadi, S. Sohrabpour, Journal of the Mechanical Behavior of Biomedical Materials 42 (2015) 292-310.

[23] M. Ashrafi, J. Arghavani, R. Naghdabadi, F. Auricchio, Journal of Intelligent Material Systems and Structures 27 (2016) 608-624.

[24] V.G. DeGiorgi, M.A. Qidwai, Smart Materials and Structures 11 (2002) 435-443. 
[25] L. Qiang, Y.J. Yuan, M.B. Chun, S.X. Dong, Materials Science \& Engineering A 419 (2006) 214-217.

[26] M. Maraldi, L. Molari, D. Grandi, Journal of Intelligent Material Systems and Structures 23 (2012) 1083-1092.

[27] T. El Sayed, E. Gürses, A. Siddiq, Computational Materials Science 60 (2012) 44-52.

[28] C. Greiner, S.M. Oppenheimer, D.C. Dunand, Acta Biomaterialia 1 (2005) 705-716.

[29] Y.P. Zhang, D.S. Li, X.P. Zhang, Scripta Materialia 57 (2007) 1020-1023.

[30] X.P. Zhang, H.Y. Liu, B. Yuan, Y.P. Zhang, Materials Science and Engineering A 481482 (2008) 170-173.

[31] D.S. Li, Y.P. Zhang, G. Eggeler, X.P. Zhang, Journal of Alloys and Compounds 470 (2009) L1-L5.

[32] S. Plimpton, Computational Materials Science 4 (1995) 361-364.

[33] S. Plimpton, Journal of Computational Physics 117 (1995) 1-19.

[34] S. Gur, V.R. Manga, S. Bringuier, K. Muralidharan, G.N. Frantziskonis, Computational Materials Science 133 (2017) 52-59.

[35] W.S. Lai, B.X. Liu, Journal of Physics: Condensed Matter 12 (2000) L53-L60.

[36] Y. Zhong, K. Gall, T. Zhu, Journal of Applied Physics 110 (2011) 033532.

[37] Y. Zhong, K. Gall, T. Zhu, Acta Materialia 60 (2012) 6301-6311.

[38] A. Stukowski, Modelling and Simulation in Materials Science and Engineering 18 (2010) 015012.

[39] N. Zotov, V. Marzynkevitsch, E.J. Mittemeijer, Journal of Alloys and Compounds 616 (2014) 385-393.

[40] Y. Zhao, M. Taya, Y. Kang, A. Kawasaki, Acta Materialia 53 (2005) 337-343.

[41] G. Maîtrejean, P. Terriault, V. Brailovski, Computational Materials Science 77 (2013) 93-101. 


\title{
Supplementary material
}

\section{Atomistic simulation of shape memory effect (SME) and superelasticity (SE) in nano-porous NiTi shape memory alloy (SMA)}

\author{
Sourav Gur ${ }^{1}$, George N. Frantziskonis ${ }^{1,2, *}$ and Krishna Muralidharan ${ }^{2}$ \\ ${ }^{1}$ Civil Engineering and Engineering Mechanics, University of Arizona, Tucson, AZ 85721 USA \\ 2 Materials Science and Engineering, University of Arizona, Tucson, AZ 85721 USA \\ * Corresponding author Email: frantzis@email.arizona.edu (G. N. Frantziskonis)
}

\section{Interatomic potential}

According to this potential, the total energy of the system over all atoms is expressed as

$$
E=\sum_{i}\left\{\sum_{j \neq i} A_{\alpha \beta} \exp \left[-p_{\alpha \beta}\left(\frac{r_{i j}}{d_{\alpha \beta}}-1\right)\right]-\sqrt{\sum_{j \neq i} F\left(r_{i j}\right)}\right\}
$$

where the first term indicates the pairwise interaction energy and the second term represents the many-body effects. Recently, the many-body interaction part (second term in (1.1)) of Lai and Liu potential has been modified by Zhong et al, such that the second terms in (11.) are expressed through the following set of equations

$$
F\left(r_{i j}\right)= \begin{cases}\xi_{\alpha \beta}^{2} \exp \left[-2 q_{\alpha \beta}\left(\frac{r_{i j}}{d_{\alpha \beta}}-1\right)\right] & r_{i j} \leq r_{1} \\ c_{0, \alpha \beta}+c_{1, \alpha \beta}\left(r_{i j}-r_{1}\right)+c_{2, \alpha \beta}\left(r_{i j}-r_{1}\right)^{2}+c_{3, \alpha \beta}\left(r_{i j}-r_{1}\right)^{3} & r_{1} \leq r_{i j} \leq r_{c}\end{cases}
$$

where $i$ and $j$ denote atoms, $\alpha, \beta$ denote the type of atoms (i.e., $\mathrm{Ni}$ or $\mathrm{Ti}$, respectively), $r_{1}$ the cutoff radii $\left(r_{c}\right)$, adopted $4.0 \AA$ and $4.2 \AA$, respectively. Different parameters of this potential are determined through fitting (i.e., lattice parameters, cohesive energy, elastic constants and vacancy formation energy) of the B2 phase at $0 \mathrm{~K}$ temperature, from first principles calculations. More details about this EAM potential parameters are provided in Zhong et al study.

\section{Structural characterization}

To distinguish austenite B2 phase from the different martensite B19' phase variants, the bond length based order parameter proposed by Mutter and Nielaba was adopted. According to this order parameter, a pristine B2 and B19' structure shows the values (like delta function) at -1.0 and +1.0 , respectively. This order parameter is expressed as

$$
\chi\left(d_{0}, d_{1}\right)=\frac{\left[d_{0}\left\{d^{(B 2)}+d_{1}^{\left(B 19^{\prime}\right)}\right\}\right]-\left[d_{1}\left\{d^{(B 2)}+d_{0}^{\left(B 19^{\prime}\right)}\right\}\right]}{\left[d^{(B 2)}\left\{d_{0}^{\left(B 19^{\prime}\right)}-d_{1}^{\left(B 19^{\prime}\right)}\right\}\right]}
$$

where different bond length parameter of pure austenite phase and martensite phase are $d^{(B 2)}=$ $2.62 \AA, d_{0}^{\left(B 19^{\prime}\right)}=2.46 \AA$ and $d_{1}^{\left(B 19^{\prime}\right)}=2.65 \AA$. The value of this order parameter becomes +1.0 
for the martensite B19' phase when $d_{0}=d_{0}^{\left(B 19^{\prime}\right)}$ and $d_{1}=d_{1}^{\left(B 19^{\prime}\right)}$. For the austenite B2 phase $d_{0}=d_{1}=d^{(B 2)}$ and thus the value of this order parameter becomes -1.0 . For the B19 phase, based on the Ni-Ti bond length, this order parameter shows approximate values either -0.16 or +0.16 (for $d_{0}=d_{0}^{(B 19)}=2.54 \AA$ and $d_{1}=d_{1}^{(B 19)}=2.69 \AA$ ). However, the lattice parameters of different phases change because of the linear thermal expansion or contraction $(\alpha \Delta T)$, and because of the internal strains $\left(\varepsilon^{*}\right)$ that develop during the phase transformation process. Thus the order parameter shows a narrow Gaussian distribution around -1.0 and +1.0 for the B2 and B19' phases, respectively.

\section{Shape Memory Effect (SME)}

Table-S1: Variation of different model parameters with respect to porosity

\begin{tabular}{|c|c|c|}
\hline Porosity (\%) & $\beta$ & $v$ \\
\hline 0.0 & 0.1159 & 2.3478 \\
\hline 5.0 & 0.1149 & 2.3155 \\
\hline 10.0 & 0.1137 & 2.1872 \\
\hline 15.0 & 0.1124 & 2.0584 \\
\hline 20.0 & 0.1115 & 1.9048 \\
\hline 25.0 & 0.1105 & 1.7538 \\
\hline 30.0 & 0.1094 & 1.6251 \\
\hline 35.0 & 0.1086 & 1.4951 \\
\hline 40.0 & 0.1073 & 1.3907 \\
\hline
\end{tabular}

\section{Superelasticity (SE)}

Table-S2: Variation of the parameters entering the linear model for phase transformation (PT) stress as a function of temperature

\begin{tabular}{|c|c|c|c|c|c|}
\hline Temperature (K) & 250 & 300 & 350 & 400 & 450 \\
\hline$\beta_{\mathrm{s}}^{\mathrm{AM}}(\mathrm{GPa})$ & 0.37143 & 0.42719 & 0.46381 & 0.49919 & 0.52207 \\
\hline$\alpha_{\mathrm{s}}^{\mathrm{AM}}(\mathrm{GPa} / \%)$ & -0.00198 & -0.00352 & -0.00463 & -0.00539 & -0.00648 \\
\hline$\beta_{\mathrm{f}}^{\mathrm{AM}}(\mathrm{GPa})$ & 0.78608 & 0.91813 & 1.24889 & 1.92327 & 2.76267 \\
\hline$\alpha_{\mathrm{f}}^{\mathrm{AM}}(\mathrm{GPa} / \%)$ & -0.00203 & -0.00447 & -0.00584 & -0.00818 & -0.01073 \\
\hline$\beta_{\mathrm{s}}^{\mathrm{MA}}(\mathrm{GPa})$ & 0.24103 & 0.31322 & 0.54844 & 0.90670 & 1.36235 \\
\hline$\alpha_{\mathrm{s}}^{\mathrm{MA}}(\mathrm{GPa} / \%)$ & -0.00198 & -0.00309 & -0.00425 & -0.00623 & -0.00872 \\
\hline$\beta_{\mathrm{f}}^{\mathrm{MA}}(\mathrm{GPa})$ & 0.07748 & 0.109941 & 0.15399 & 0.21206 & 0.27051 \\
\hline$\alpha_{\mathrm{f}}^{\mathrm{MA}}(\mathrm{GPa} / \%)$ & -0.00141 & -0.00192 & -0.00235 & -0.00326 & -0.00381 \\
\hline
\end{tabular}


Table-S3: Variation of the parameters entering the exponential model for esidual strain, phase transformation (PT) strain, and normalized dissipated energy as a function of temperature

\begin{tabular}{|c|c|c|c|c|c|}
\hline Temperature (K) & 250 & 300 & 350 & 400 & 450 \\
\hline$\beta_{\mathrm{r}}(\%)$ & 1.21435 & 0.66529 & 0.14824 & 0.08130 & 0.01154 \\
\hline$\alpha_{\mathrm{r}}(1 / \%)$ & 0.01462 & 0.02013 & 0.04349 & 0.03972 & 0.04979 \\
\hline$\beta_{\mathrm{l}}(\%)$ & 3.44592 & 2.53862 & 2.32962 & 1.90933 & 1.76773 \\
\hline$\alpha_{\mathrm{l}}(1 / \%)$ & 0.01113 & 0.01789 & 0.02154 & 0.26504 & 0.31771 \\
\hline$\alpha_{\mathrm{n}}(1 / \%)$ & 0.01505 & 0.01879 & 0.02368 & 0.02722 & 0.03091 \\
\hline
\end{tabular}

